Background HIV infection is associated with increased risk of cancer:

1. AIDS-defining cancers (ADC): Kaposi's sarcoma (KS), nonHodgkin lymphoma (NHL), cervix cancer.

2. non-AIDS-defining cancers (NADC): Hodgkin lymphoma (HL), anal cancer, lung, head, neck, hepatocarcinoma.

Purpose To analyse patients with antiretroviral therapy and chemotherapy, type of cancer and associated risk factors.

Materials and Methods Descriptive study of patients with antiretroviral and chemotherapy between 2004-2011, extracting data from medical records and the Farmatools programme, analysing using SPSS 11.0

Results 33 patients were obtained (3.7\% of all HIV patients on antiretroviral treatment); $82 \%$ men: 16 with ADC (11 NHL, $3 \mathrm{KS}$, and 2 with NHL and KS) and 17 with NADC (5 HL, 3 lung cancer, 3 head-neck, 3 anal, 1 ovary, 1 gastric and 1 chronic lymphocytic leukaemia). When cancer was diagnosed patients presented: CD4 $<200$ cells/microliter (27.3\%), detectable viral load (VL) (33.3\%), C3 category (63.6\%), smokers $(63.6 \%)$, human papillomavirus (HPV) (6.1\%), Epstein Barr virus (21.2\%), human herpes virus 8 (HHV8)(21.2\%), hepatitis B-C (48.5\%), intravenous drug addict (24.2\%). 8 patients died.

$80 \% \mathrm{KS}$ patients and $66.7 \%$ head-neck cancer had CD $4<200$ $(\mathrm{P}=0.036) .62 .5 \%$ of those who died presented $\mathrm{CD} 4<200$ $(\mathrm{P}=0.009) .66 .6 \%$ of anal cancer patients presented HPV $(\mathrm{P}=0.006) .100 \%$ of KS presented HHV8 ( $\mathrm{P}=0.002)$.

Conclusions $3.7 \%$ of HIV patients on treatment developed neoplasms, more than $50 \%$ were NADC, of which $88 \%$ started in patients with an undetectable VL, confirming a nice immunological status when cancer was diagnosed.

No conflict of interest.

\section{CPC-152 USE OF OMALIZUMAB IN CHRONIC COLD URTICARIA: A CASE REPORT}

doi:10.1136/ejhpharm-2013-000276.609

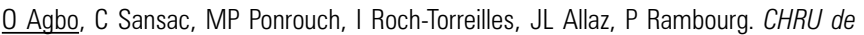
Montpellier, hérault, Montpellier, France

Background Omalizumab is a recombinant humanised monoclonal antibody which prevents the binding of IgE to the high-affinity receptor type I (FcERI). A complicated series of reactions results in a reduction of free IgE responsible for the allergic inflammatory cascade. Omalizumab is indicated as add-on therapy to improve asthma control in adults and adolescents (from 12 years). In addition, several studies show that omalizumab is effective in the treatment of chronic urticaria.

Purpose We report the case of a patient with chronic cold urticaria resistant to conventional treatments.

Materials and Methods The patient was a 67-year-old man, who had suffered from chronic urticaria for over 30 years. The disease was disclosed by pressure urticaria, which had been neglected for a long time. It then turned into a cold urticaria in the $90 \mathrm{~s}$. The latter showed itself in 2002 as the patient experienced an anaphylactic shock in a bath at $24^{\circ} \mathrm{C}$.

Results Several lines of treatment, all unsuccessful, were tested on the patient: high-dose $\mathrm{H} 1$ antihistamine, montelukast, methotrexate, anakinra. In view of this therapeutic impasse, omalizumab appeared as an alternative: doses of $375 \mathrm{mg}$ were administered to him every 15 days as a start. In total, 12 treatments were performed in dermatology outpatients. No side effects were encountered except for an episode of nausea. The results were: a decrease in consumption of $\mathrm{H} 1$ antihistamine, ice test negative and significant clinical improvement of his urticaria.

Conclusions In view of the results obtained for this patient, omalizumab appears to be an alternative for treating chronic urticaria in treatment failure. Indeed, it is well tolerated, the risk-benefit ratio is positive, the only problem is the cost incurred for such care.

No conflict of interest.

\section{CPC-153 WARD PHARMACIST: MANAGING INTERACTIONS IN THE DEPARTMENT OF HAEMATOLOGY AND BONE MARROW TRANSPLANTATION}

doi:10.1136/ejhpharm-2013-000276.610

G Saibene, F Brera, E Togliardi, G Antonacci, F Festinese, M Mazzer, V Di Mauro. Fondazione IRCCS Istituto Nazionale Dei Tumori, Farmacia, Milano, Italy

Background The Milan National Cancer Institute Pharmacy began a collaboration with the haematology and bone marrow transplantation (ETMO) department, to optimise concomitant conditioning protocols of bone marrow transplants; the pharmacokinetics and pharmacodynamics are affected by the high doses of chemotherapy administered. The drugs analysed were those in the conditioning schedules used in accordance with international guidelines.

Purpose To provide a practical guide for managing drug interactions between the drugs commonly used by ETMO and those in the transplant conditioning schedules.

Materials and Methods The presence of the ward pharmacist, funded by the Italian Haematology Society, allowed the daily management of treatment to be investigated. Databases were used (Micromedex, Codifa) and literature meta-analyses were conducted, in order to obtain the pharmacokinetic and pharmacodynamic characteristics of these drugs and possible interactions.

Results Within our Institute, 72 transplants that used conditioning were performed in a year, 32 autologous and 40 allogeneic. In particular, 28 transplants used a high-dose melphalan scheme, 28 used thiotepa/fludarabine/cyclophosphamide, 4 used BEAM, 4 used TBI/fludarabine/cyclophosphamide and 8 used the KROGER scheme. Therefore the interactions between drugs used in the protocols themselves and the drugs commonly used within the department by transplant patients were analysed. For this purpose the following drugs were considered: ciclosporin, allopurinol, acetazolamide and IPP. Following this analysis, it was shown that there were significant interactions between the drugs used in the conditioning scheme and drugs commonly used in patients with bone marrow transplants.

Conclusions The pharmacist set up a means of enabling a clinician to browse for a more informed choice: dedicated schemes are being developed, in which they report any interactions observed, associated with the treatment protocols. All this has therefore contributed to the rational use of the drugs and resources, for example the use of antifungals after transplantation and not before, and the introduction of pantoprazole instead of omeprazole. A future goal will be the analysis of the interactions between the drugs and concomitant haematology chemotherapy.

No conflict of interest.

\section{International posters}

\section{INT-009 POINT PREVALENCE STUDIES ON ANTIBIOTIC USAGE AT THE CHILDREN'S UNIVERSITY HOSPITAL}

doi:10.1136/ejhpharm-2013-000276.611

I Sviestina, D Mozgis. Children's University Hospital in Riga, Latvia,

Background Due to higher use of broad spectrum agents in the treatment of both adults and children, hospitals are considered to be centres of antimicrobial resistance. According to several studies, approximately $60 \%$ of hospitalised children will receive at least one antibiotic. 